\title{
Impulsive Noise Models Used in Power Line Communications
}

\author{
S. M. ÇÜRÜK
}

\begin{abstract}
In a communication system, noise has always been considered as a serious cause of error. Although most common noises can be modeled by well-known Gaussian distributions, some communication systems experience other types of noise. Power Line Communication (PLC) is one of these systems which run on a difficult medium since it uses the preexisting power line network for transmission. PLC experiences several different types of human-made and natural noise which are mostly impulsive and cannot be modeled simply by Gaussian. Different statistical models are used for characterizing the impulsive noise of PLC systems in the literature. The purpose of this study is to go over the impulsive noise models previously presented in order to have a contribution to this hot topic. We point out the similarities and differences of the models, namely Middleton Class A, Bernoulli Gaussian and Alpha Stable statistical models. It is presented that all have heavy tails which make them appropriate for impulsive noise. Although Middleton Class A is more generic, Bernoulli Gaussian model is wide enough and can be preferable because of its compact simple form especially when analytical results are needed.
\end{abstract}

Index Terms - Alpha Stable distribution, Bernoulli Gaussian distribution, Impulsive Noise, Middleton Class A distribution.

\section{INTRODUCTION}

$\mathrm{T}$ HE noise is the main reason of errors occurred in data transmission and should be dealt with carefully for reliable communication. The common noise experienced in a communication system is the thermal noise and it can easily be modeled by a Gaussian distribution. Unfortunately, the effects of various human-made and/or natural noises are also observed in some communication systems, which are mostly impulsive and cannot be modeled by Gaussian density. A Power Line Communication (PLC) system is one of these systems.

PLC shares the installed power lines with various electrical sources for data transfer. Unfortunately, the power line medium is unsafe for communication and various noise types may be experienced. These noises are named as colored

SELVA MURATOĞLU ÇÜRÜK, is with Department of Electrical and Electronics Engineering of Iskenderun Technical University, Hatay, Turkey, (e-mail: selva.curuk@iste.edu.tr).

(iD) https://orcid.org/ 0000-0002-2195-7827 background noise, narrowband noise, periodic asynchronous/synchronous impulsive noise and asynchronous impulsive noise [1]. Colored noise and narrowband noise are background noise and are modeled as Gaussian; but the rests are all impulsive and should be modeled exhaustively. The human-made noises such as switching (connection and removal of the sources) from the network, sudden power variations (changing speed of an ac motor etc.) or natural noises such as electromagnetic interference are all causes of impulsive noise in PLC. Impulsive noise is the more feared one because its prediction is not easy and its level could even be higher than the background noise.

For safe PLC, accurate models with true characteristics of impulsive noise are needed. Researches modeling the impulsive noise in different fields of communications, as well as in PLC, are present in literature. According to [1], the statistical properties of impulsive noise in a PLC system fit Alpha Stable distribution. The article [2] points out some features of impulse noise models, Middleton Class A, Bernoulli-Gaussian and Symmetric Alpha Stable. In [3] a new approach is applied to modeling of impulsive noise at the sources. In [4], the generalized Gaussian distribution is introduced as a suitable model after analyzing noise amplitude. [5] and [6] are interested in coping with impulsive noise which is modeled by Bernoulli Gaussian distribution. The authors in [7] used Middleton Class A noise model in their studies. Authors of [8] search the suitability of the Middleton Class A model for narrowband PLC noise. Frequency domain background and impulsive noises for broadband PLC systems are characterized in [9]. The authors of [10] compare Middleton Class A and Marcov-Middleton models. The authors of [11] modeled power line colored background noise, narrowband noise and burst noise. A simple mathematical representation of time variant and nonwhite noise for narrowband PLC is introduced in [12]. The authors in [13] give an easily implemented noise model for power line channel. Papers [14] and [15] are investigating statistical noise models.

We see it necessary to bring a general view of impulse noise models used in PLCs in order to sum up. A comparative study of the common models seen in the literature, namely Middleton Class A, Bernoulli Gaussian and Alpha Stable statistical models is given in the following sections. 


\section{IMPULSIVE NOISE MODELS}

In a communication system, impulsive noise is categorized into two groups: Human-made impulsive noise is caused by different sources, such as switching, dropouts or degradation of data, while naturally occurring impulsive noise is due to atmospheric phenomena and solar static. The human-made impulsive noise is described as trains of non-overlapping pulses while the impulsive noise due to nature is the random superposition of the pulses formed with the effects of the natural phenomena. Although impulsive noise duration is more than one sample long, it is relatively short compared to transmission.

The first detailed model of impulsive noise was given by Middleton [2], where impulsive noise was defined as randomly occurring sequences of pulses or impulses in time, with varying duration and intensity. Thus, in Middleton's model impulsive noise is a non-stationary, binary-state sequence of impulses with random amplitudes and random occurrence positions. Following, various studies have been done to fit the first order statistics of the noise to the known distributions, where Symmetric Alpha Stable and Bernoulli Gaussian models are the more featured ones in the literature. In this section, we examine the most popular models which appear more frequently in the literature for impulsive noise modeling.

\section{A. Gaussian Mixture Model}

In a complex data, several different regions may be observed, some with high probabilities whereas the others with smaller probabilities. Such a multimodal data is modeled in terms of a mixture of several components, where each component has a simple parametric form, such as Gaussian. Thus, it is assumed that each data point belongs to one of the components and the distribution for each component is assigned separately.

A Gaussian Mixture model assumes all data are generated from a mixture of a finite number of Gaussian distributions with unknown parameters. According to this probabilistic model, random variable $n_{k}$ has a distribution where the probability density function (pdf) is a weighted summation of different Gaussian distributions [7], i.e.,

$$
p\left(n_{k}\right)=\sum_{m=0}^{K} P_{m} . \aleph\left(n_{k} ; \mu_{m}, \sigma_{m}{ }^{2}\right)
$$

$\aleph\left(n_{k} ; \mu_{m}, \sigma_{m}{ }^{2}\right)$ denotes Gaussian pdf with mean $\mu_{m}$, variance $\sigma_{m}^{2}$ and $P_{m}$ is mixing probability of the $m$-th Gaussian component which sums to 1 , i.e.,

$$
\sum_{m=0}^{K} P_{m}=1, \quad 0<P_{m}<1
$$

Gaussian Mixture is the base of various distributions; Middleton Class A and Bernoulli Gaussian models are among them.

\section{B. Middleton Class A Model}

Middleton has defined three statistical models depending on the bandwidth of impulsive noise and receiver and they have been widely used in electromagnetic and communication problems. (Class A noise has narrower bandwidth than that of the receiver; while Class $\mathrm{B}$ noise has larger bandwidth; and Class $\mathrm{C}$ noise is the sum of both noises.) In communication systems, as in PLC, Middleton Class A noise model is employed more widely compared to other two classes.

In Middleton Class A model, $L$, number of impulses in an observation period $T_{0}$, is assumed to obey a Poisson distribution. i.e., this model is a form of the Poison model, with the impulse width is taken into account [2]:

$$
P_{T_{0}}(L)=\frac{\left(\eta \cdot T_{0}\right)^{m} \cdot e^{-\left(\eta \cdot T_{0}\right)}}{L !}
$$

where $\eta$ is the average number of impulses per second $\left(\eta \cdot T_{0}\right.$ is the average number of impulses in the period $T_{0}$ ). Then, the pdf of a Middleton Class A noise sample $n_{k}$ is given as follows:

$$
\begin{gathered}
p\left(n_{k}\right)=\sum_{m=0}^{\infty} P_{m} \cdot \aleph\left(n_{k} ; 0, \sigma_{m}{ }^{2}\right) \\
P_{m}=\frac{A^{m} \cdot e^{-A}}{m !}
\end{gathered}
$$

The non-Gaussian nature of the noise is measured by the parameter $A$. Impulsive index (non-structure index) $A$ is the density of impulses in the observation period and is in the range $A \in(0, \infty)$. Lower $A$ values mean fewer number of events, where the individual events dominate the instantaneous noise properties. As $A$ gets larger, the noise becomes less structured and the statistics approach the Gaussian distribution as stated in Central Limit Theorem.

The Gaussian pdf $\aleph\left(n_{k} ; 0, \sigma_{m}{ }^{2}\right)$ in (4) are zero mean with variance $\sigma_{m}^{2}$ which is given by

$$
{\sigma_{m}}^{2}=\sigma_{I}^{2} \cdot \frac{m}{A}+\sigma_{g}^{2}
$$

$\sigma_{I}^{2}$ is the variance of the impulse noise and $\sigma_{g}^{2}$ is the variance of the background Gaussian noise. The $m$-th variance can be written equivalently as 


$$
\sigma_{m}^{2}=\sigma_{g}^{2} \cdot\left(\frac{m}{A . \Gamma}+1\right)=\sigma^{2} \cdot\left(\frac{m / A+\Gamma}{1+\Gamma}\right)
$$

The parameter $\Gamma=\sigma_{g}{ }^{2} / \sigma_{I}{ }^{2}$ is called the Gaussian factor and gives Gaussian to impulse noise power ratio. $\sigma^{2}=\sigma_{g}^{2}+\sigma_{I}^{2}$ is the total power of noise. By adjusting the parameters $A$ and $\Gamma$, the Middleton Class A density can cover a great variety of non-Gaussian noise densities. It would be noted that for an impulsive noise in PLC, generally $\Gamma$ is in the range of $\left[10^{-6}, 1\right]$ and $A \in\left[10^{-2}, 1\right][10]$.

\section{Bernoulli Gaussian Model}

Another popular impulsive noise model is the Bernoulli Gaussian model. According to this model, the overall noise sample $n_{k}$ may be expressed as [16]:

$$
n_{k}=w_{k}+i_{k}
$$

where $w_{k}$ is the white Gaussian background noise and $i_{k}$ is the impulsive noise sample given by

$$
i_{k}=b_{k} \cdot g_{k}
$$

$g_{k}$ is complex white Gaussian noise with mean zero and $b_{k}$ is the Bernoulli process which means the arrival of impulsive noise has a probability

$$
P\left(b_{k}\right)=\left\{\begin{array}{cc}
p, & b_{k}=1 \\
1-p, & b_{k}=0
\end{array}\right.
$$

In (10), $p$ represents the impulsive bursts probability of occurrence.

The Bernoulli Gaussian noise model is described by the following pdf:

$$
p\left(n_{k}\right)=(1-p) . \aleph\left(n_{k} ; 0, \sigma_{g}^{2}\right)+p . \aleph\left(n_{k} ; 0, \sigma_{g}{ }^{2}+\sigma_{I}^{2}\right)
$$

where $\aleph\left(n_{k} ; 0, \sigma_{m}{ }^{2}\right)$ represents the Gaussian pdf. Bernoulli Gaussian noise model is a Gaussian Mixture with two terms.

\section{Alpha Stable Model}

The noise models discussed above are the most widely used ones, but Symmetric Alpha Stable distribution is also used for impulsive noise modeling in the literature. Central Limit Theorem states that, the normalized sum of independent and identically distributed random variables with finite variance converges to the Gaussian distribution as the number goes to infinity. Generalized Central Limit Theorem underlines that the sum converges to Alpha Stable distribution when the finite variance limitation (it may be finite or infinite variance) drops. Alpha Stable distributions date back, but they have a limited usage because except for a few cases their pdfs cannot be expressed in closed-forms. Nowadays Alpha Stable distributions are being used more, as its usage in PLC noise models, since computation with powerful computers is possible.

An Alpha Stable random variable is defined by its characteristic function [1]:

$\phi(u)= \begin{cases}\exp \left\{j \cdot \delta \cdot u-\gamma^{\alpha} \cdot|u|^{\alpha} \cdot\left(1+j \cdot \beta \cdot \operatorname{sign}(u) \cdot \tan \left(\frac{\pi \cdot \alpha}{2}\right)\right)\right\}, & \alpha \neq 1 \\ \exp \left\{j \cdot \delta \cdot u-\gamma \cdot|u| \cdot\left(1-j \cdot \beta \cdot \frac{2}{\pi} \cdot \operatorname{sign}(u) \cdot \ln |u|\right)\right), & \alpha=1\end{cases}$

where the sign function is defined as

$$
\operatorname{sign}(u)=\left\{\begin{array}{cc}
1, & u>0 \\
0, & u=0 \\
-1, & u<0
\end{array}\right.
$$

Stability index $\alpha$, which is in the range of $(0,2]$, is the characteristic exponent and controls the tail decays. Skewness parameter $\beta$ takes on the values $-1 \leq \beta \leq 1$; 0 for a symmetrical distribution, $\beta>0$ for right-skewed distributions and $\beta<0$ for left-skewed ones. Finally, scale parameter $\gamma>0$ is used to scale the random variable and shift parameter $\delta \in \mathfrak{R}$ is used to control the location of the variable. The mean of an Alpha Stable distribution is $\mu=\delta-\beta \cdot \gamma \cdot \tan (\pi \cdot \alpha / 2)$ for $\alpha>1$, but undefined for $\alpha \leq 1$. Similarly, the variance of the distribution is undefined for values of $\alpha<2$ but for $\alpha=2$, the variance is $\sigma^{2}=2 \cdot \gamma^{2}$.

The Gaussian distribution is a special case of Alpha Stable distributions when $\alpha=2$. Although the value of $\beta$ has no effect in this situation, it is usually written as $\beta=0$. The other special cases are Cauchy distribution with $\alpha=1, \beta=0$ and Lévy distribution with $\alpha=0.5, \beta=1$. Except for the Gaussian case, Alpha Stable distribution tail decay is slow and therefore they are considered for modeling of impulsive noise [1].

\section{Simulation ResUlts}

Middleton Class A density can cover a great variety of densities by arranging its parameters. Fig. 1 gives the distribution pdfs for $\Gamma=0.01$ and various $A$ values. For the sake of simplicity, in the simulations we will consider signals with total power 1. i.e., $\sigma^{2}=\sigma_{g}{ }^{2}+\sigma_{I}{ }^{2}=1$, and this term will be omitted in (7). The number of terms in summation is selected to be high enough. As seen from the figure, the tails 
are more pronounced compared to Gauss distribution, especially for $A=0.5$ and $A=1$.

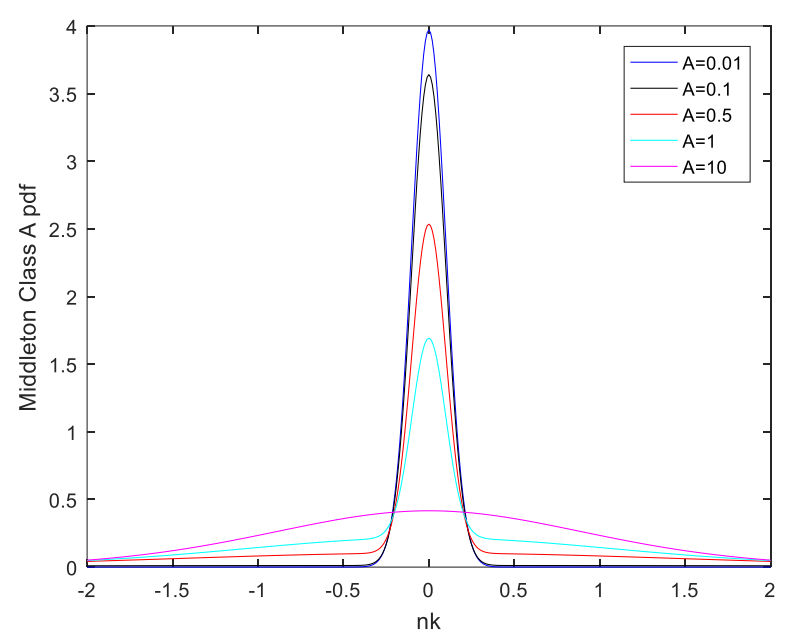

Fig. 1. Middleton Class A pdf, $\Gamma=0.01$.

Middleton Class A density is a Gaussian Mixture but theoretically with infinite summation terms. Indeed this distribution can be approximated by the first few terms for obtaining a more simplified workable form, and still it may be sufficiently accurate [10]. As seen from (5), the mixing probability $P_{m}$ decreases to zero as the term number $m$ increases, with a speed depending on the value of selected $A$. Table 1 gives mixing probability $P_{m}$ for $A=0.01, A=0.1$ and $A=1$. As it can be seen from the table, Middleton Class A pdfs can be approximated by 2 terms when $A=0.01,3$ terms when $A=0.1$ and 6 terms when $A=1$. Thus, for small $A$ values (highly impulsive case), the model may be equal to Bernoulli Gaussian model with appropriate parameter selections.

TABLE I

MIDDLETON CLASS A MODEL MIXING PROBABILITY $\left(P_{m}\right)$ VALUES FOR $A=0.01, A=0.1$ AND $A=1$

\begin{tabular}{|l|l|l|l|}
\hline \multirow{2}{*}{} & \multicolumn{3}{|l|}{$P_{m}$} \\
\cline { 2 - 4 } & $A=0.01$ & $A=0.1$ & $A=1$ \\
\hline $\mathrm{m}=0$ & 0.9900 & 0.9048 & 0.3678 \\
\hline $\mathrm{m}=1$ & 0.0099 & 0.0904 & 0.3678 \\
\hline $\mathrm{m}=2$ & 0.0000 & 0.0045 & 0.1839 \\
\hline $\mathrm{m}=3$ & 0.0000 & 0.0001 & 0.0613 \\
\hline $\mathrm{m}=4$ & 0.0000 & 0.0000 & 0.0153 \\
\hline $\mathrm{m}=5$ & 0.0000 & 0.0000 & 0.0030 \\
\hline $\mathrm{m}=6$ & 0.0000 & 0.0000 & 0.0005 \\
\hline $\mathrm{m}=7$ & 0.0000 & 0.0000 & 0.0000 \\
\hline
\end{tabular}

For an approximated Middleton Class A pdf, the summation in (4) is limited by the first $K$ terms where normalization follows, which results with a pdf given by

$$
p\left(n_{k}\right)=\sum_{m=0}^{K-1} P_{m}{ }^{\prime} \aleph\left(n_{k} ; 0, \sigma_{m}{ }^{2}\right)
$$

$$
P_{m}{ }^{\prime}=\frac{P_{m}}{\sum_{m=0}^{K-1} P_{m}}
$$

Fig. 2, Fig. 3, Fig. 4, Fig. 5 and Fig. 6 depict the pdfs of Middleton Class A distribution for several values of $A$ and $\Gamma$ in logarithmic scale. The logarithmic scale is preferred in plots to investigate the tails of the distributions thoroughly. It can be observed that when $A=10$ (Fig. 2) the resulting pdfs are almost like the Gaussian ones, as expected, and the weight of $\Gamma$ is hardly visible. There is an indistinct peak for very small $\Gamma$ values. As $A$ gets smaller, the peak becomes sharper and the tails fatter. For a fixed $A$, the value of $\Gamma$ determines the width of the shoulders. Thus as it gets larger, the pdf is wider.

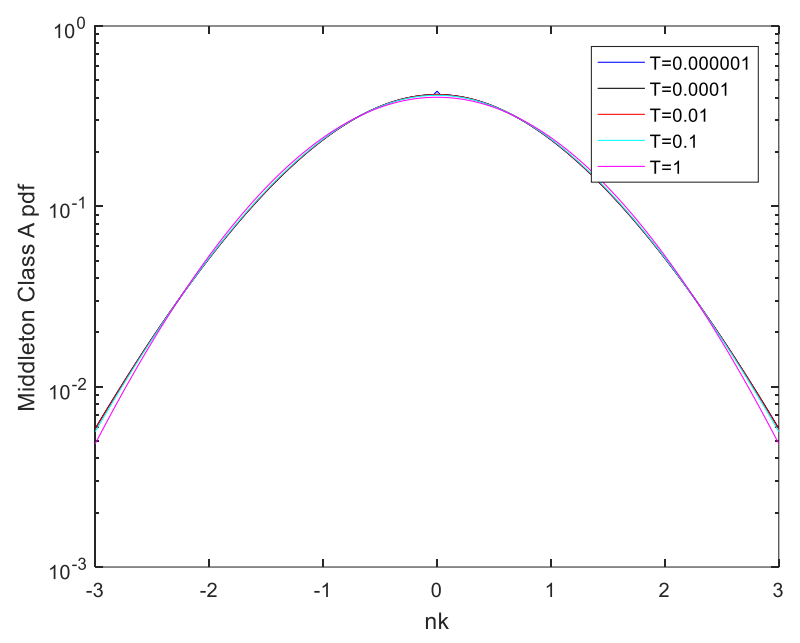

Fig. 2. Middleton Class A pdf, $A=10$.

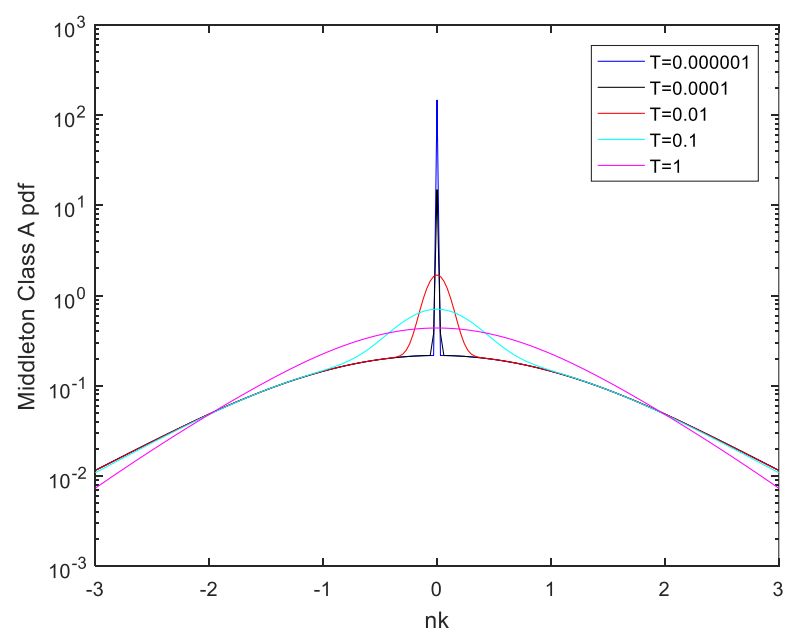

Fig. 3. Middleton Class A pdf, $A=1$. 


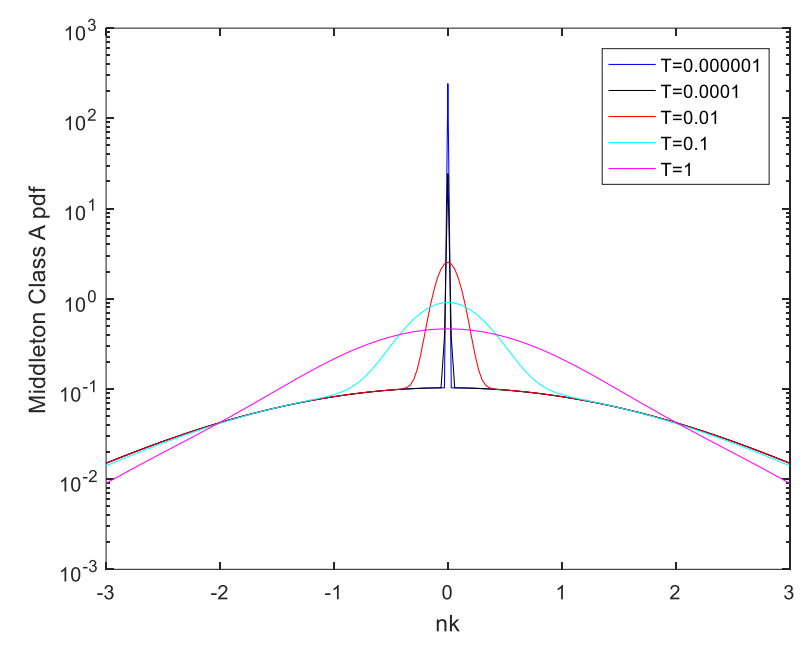

Fig. 4. Middleton Class A pdf, $A=0.5$.

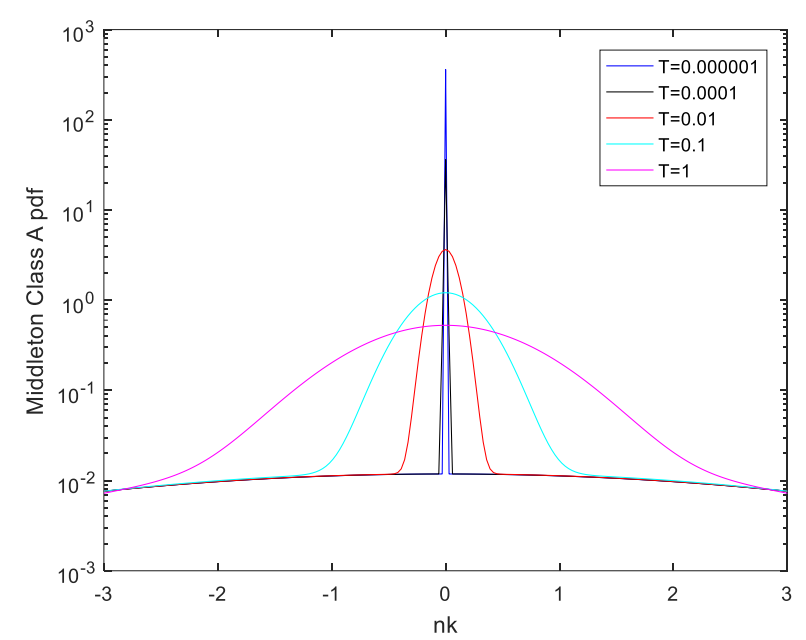

Fig. 5. Middleton Class A pdf, $A=0.1$.

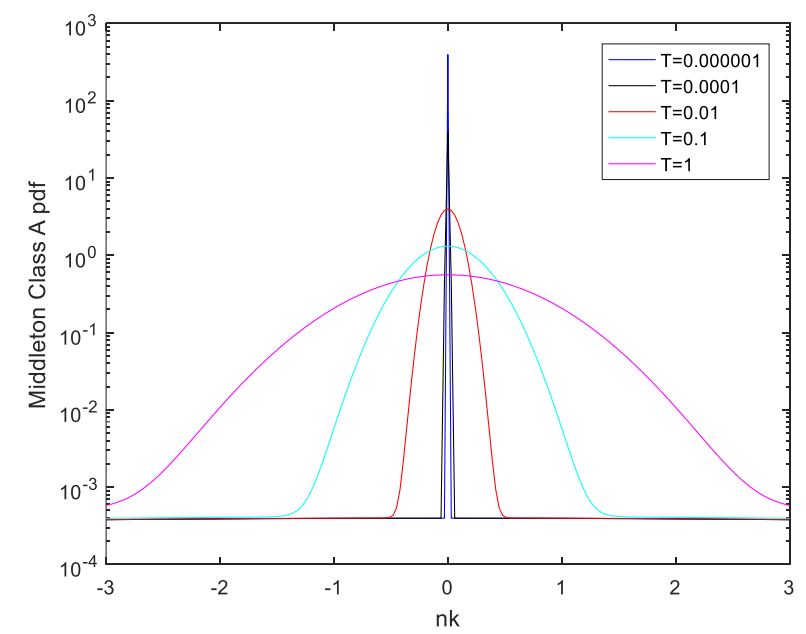

Fig. 6. Middleton Class A pdf, $A=0.01$.

The Bernoulli Gaussian noise model has been frequently used for PLC impulsive noise in the literature. Fig. 7 gives the pdfs of Bernoulli Gausian distribution for various probability $p$ and $\sigma_{g}{ }^{2}=0.1$. Again, for comparison, we consider the signals with $\sigma_{g}{ }^{2}+\sigma_{I}{ }^{2}=1$ in the simulations. As seen from the figure, similar to Middleton Class A distribution, it has heavy tails compared to Gaussian distribution, and as the probability of impulsive noise increases, the tails of pdfs become more pronounced.

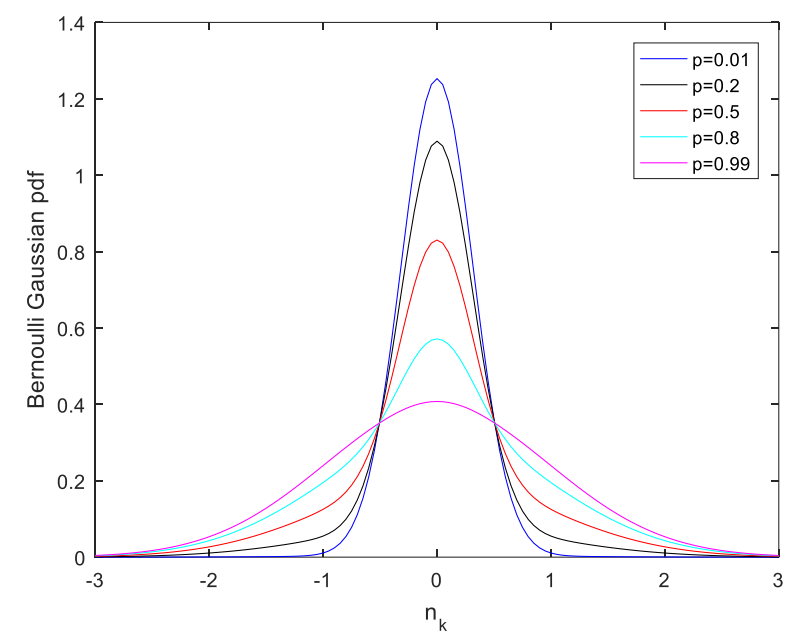

Fig. 7. Bernoulli Gaussian pdf, $\sigma_{g}^{2}=0.1$.

Fig. 8, Fig. 9, Fig. 10, Fig. 11 and Fig. 12 depict the pdfs of Bernoulli Gaussian distribution for several values of $p$ and $\sigma_{g}{ }^{2}$ in logarithmic scale to investigate the tails of the distributions. It can be observed that when $p=0.99$ (Fig. 8) the resulting pdfs are almost like the Gaussian ones, and the influence of $\sigma_{g}{ }^{2}$ is very small. As $p \rightarrow 0$, the peak becomes sharper and the tails fatter as expected. For a fixed $p$, the value of $\sigma_{g}{ }^{2}$ determines the width of the shoulders.

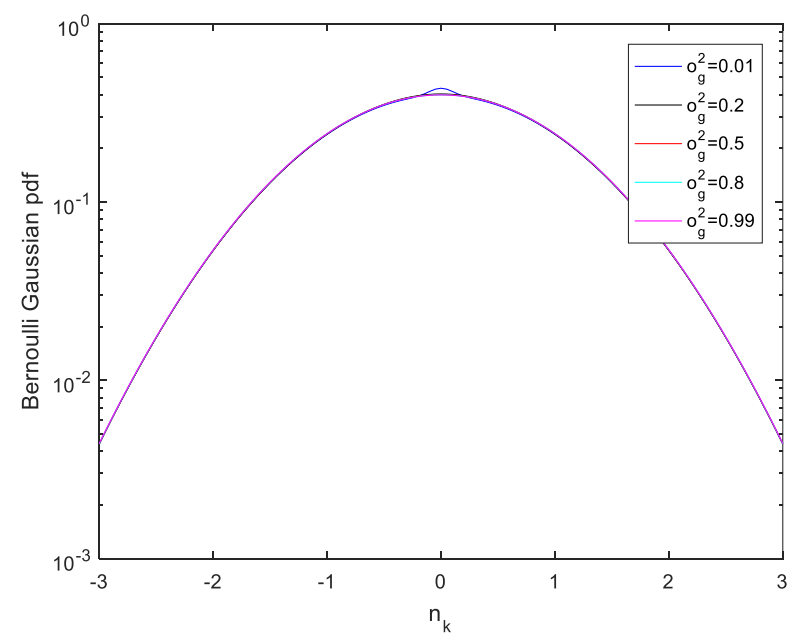

Fig. 8. Bernoulli Gaussian pdf, $p=0.99$. 


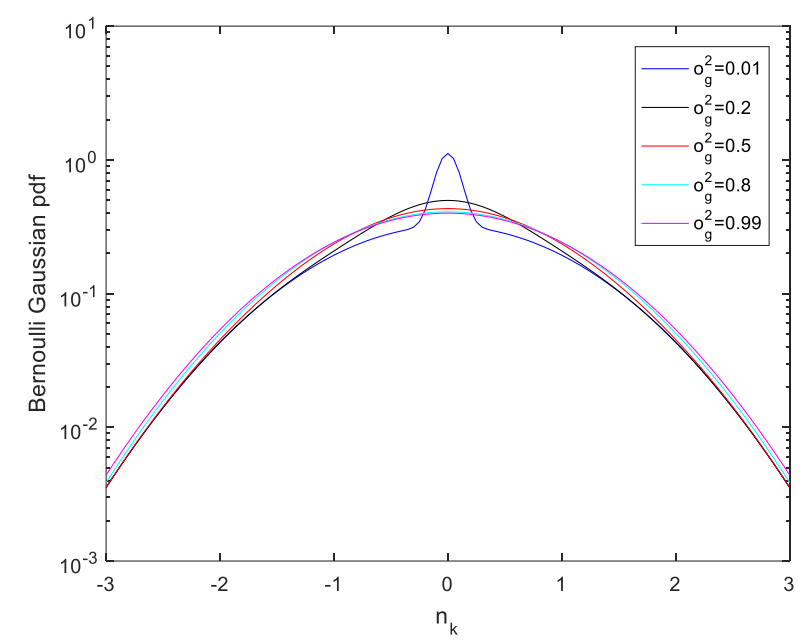

Fig. 9. Bernoulli Gaussian pdf, $p=0.8$.

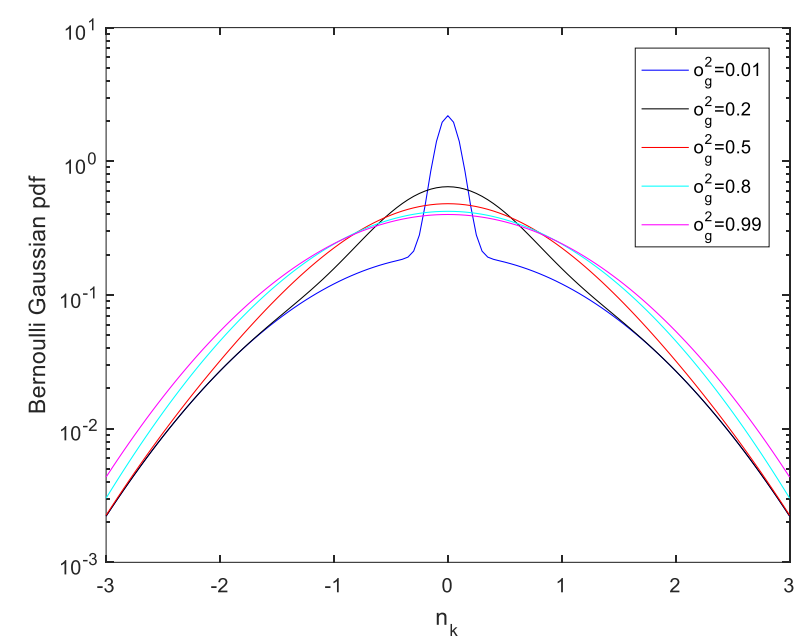

Fig. 10. Bernoulli Gaussian pdf, $p=0.5$.

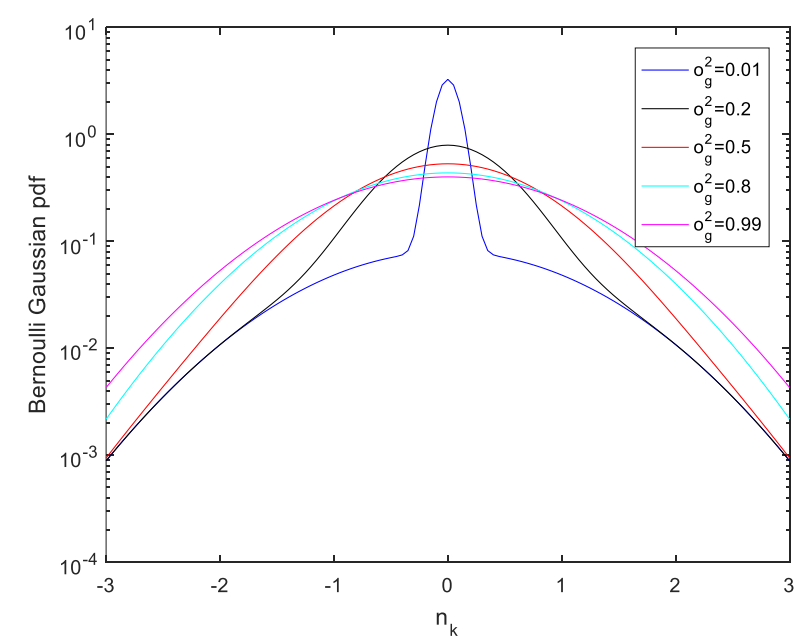

Fig. 11. Bernoulli Gaussian pdf, $p=0.2$.

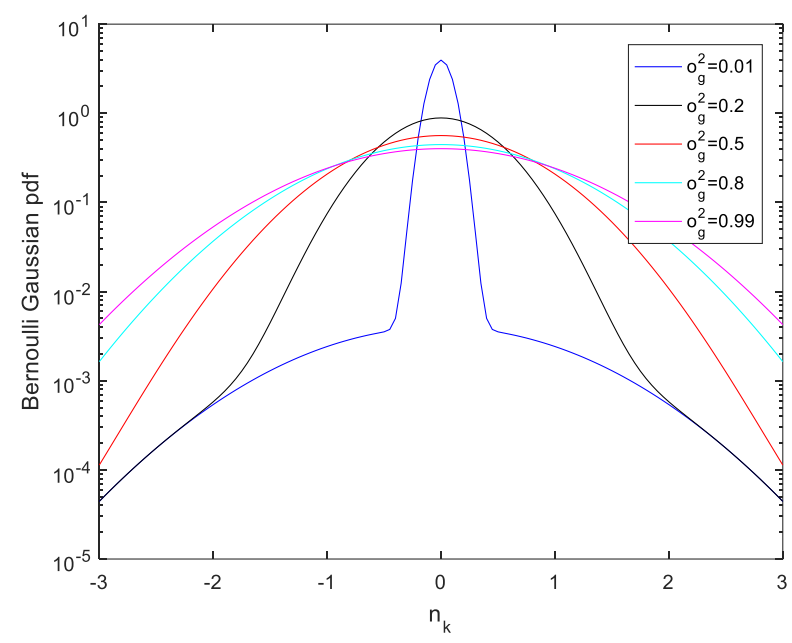

Fig. 12. Bernoulli Gaussian pdf, $p=0.01$.

Bernoulli Gaussian distributions give very close results to the Middleton Class A distributions. But the Middleton Class A model has the advantage of having its parameters directly related to the physical channel. Another point is that since it can be arranged to contain more than two Gaussian distributions, Middleton Class A model is more powerful and can model wider class of data more precisely. It can easily be adjusted to approximate even the Bernoulli Gaussian model, by adjusting the parameters. But some researchers may prefer to use the simpler one, Bernoulli Gaussian impulsive noise model rather than the Middleton Class A noise model because it is more tractable, especially when analytic results are needed.

The Alpha Stable distribution pdfs are given in Fig. 13 for various skew values $\beta$, with $\alpha=0.5, \gamma=1$ and $\delta=0$. As seen from the figure, right and left skewed distributions as well as symmetric ones can be obtained. Fig. 14, Fig. 15 and Fig. 16 give the pdfs of Symmetric Alpha Stable distributions for several values of $\alpha$ and $\gamma$ in logarithmic scale again. The lower $\alpha$ values means the heavier tails especially for small $\gamma$ values. As scale parameter gets higher, the tails are thicker after a while, for low $\alpha$ values. It should be noted that the Alpha Stable pdfs has not shoulders as in the other two distributions, Middleton Class A and Bernoulli Gaussian. 


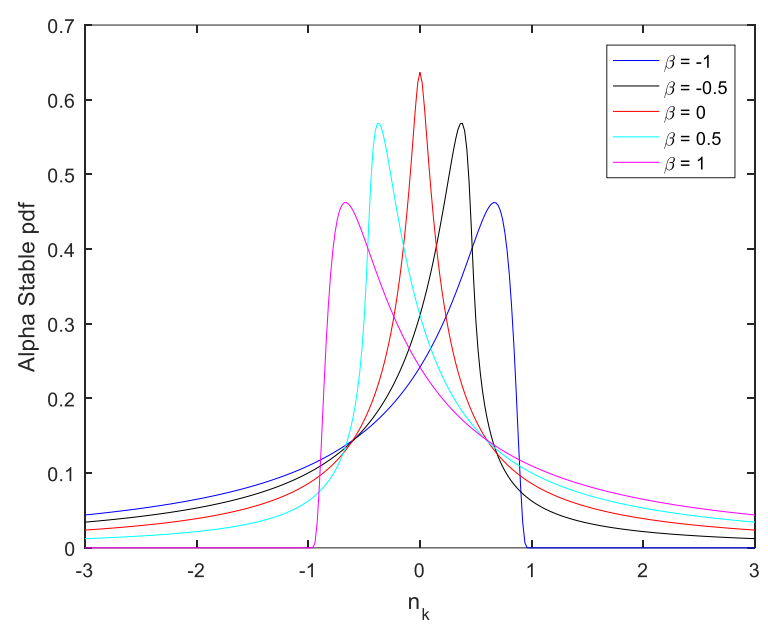

Fig. 13. Alpha stable pdf, $\alpha=0.5, \gamma=1$ and $\delta=0$.

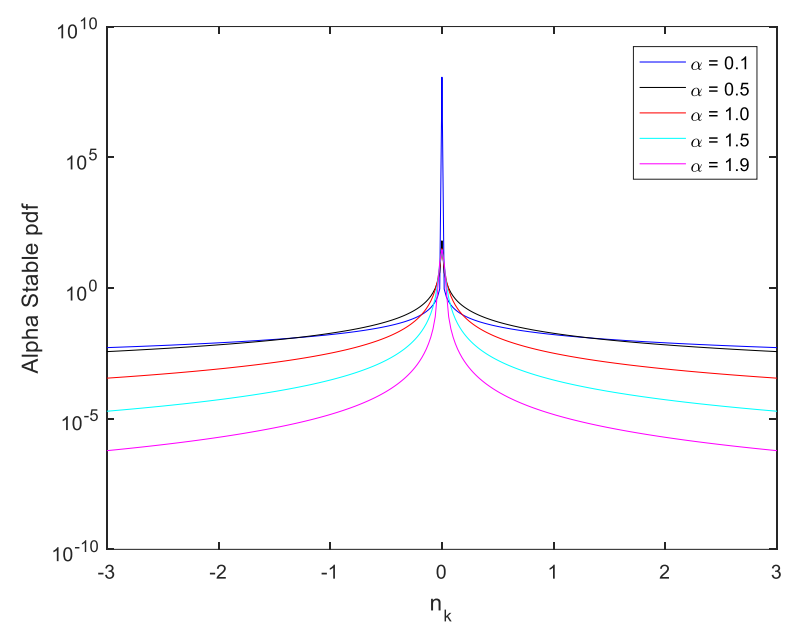

Fig. 14. Alpha stable pdf, $\beta=0, \gamma=0.01$ and $\delta=0$.

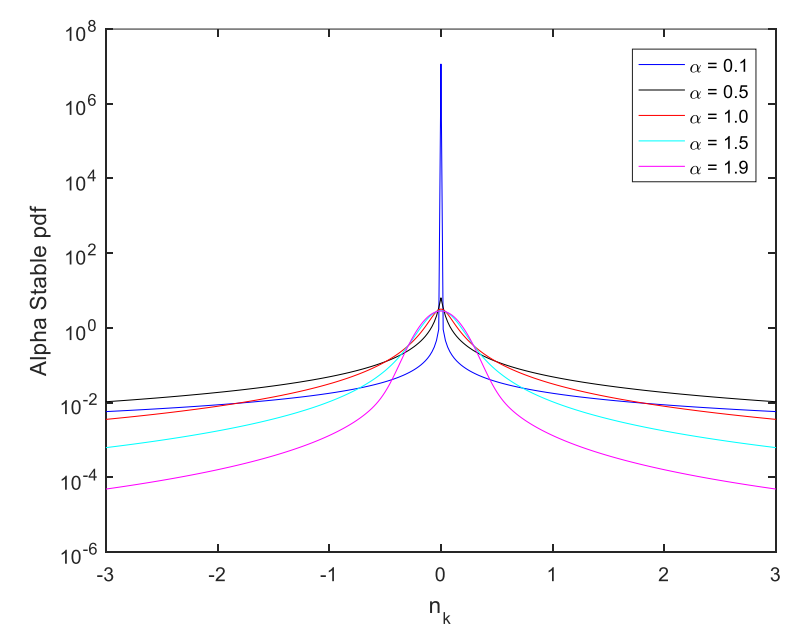

Fig. 15. Alpha stable pdf, $\beta=0, \gamma=0.1$ and $\delta=0$.

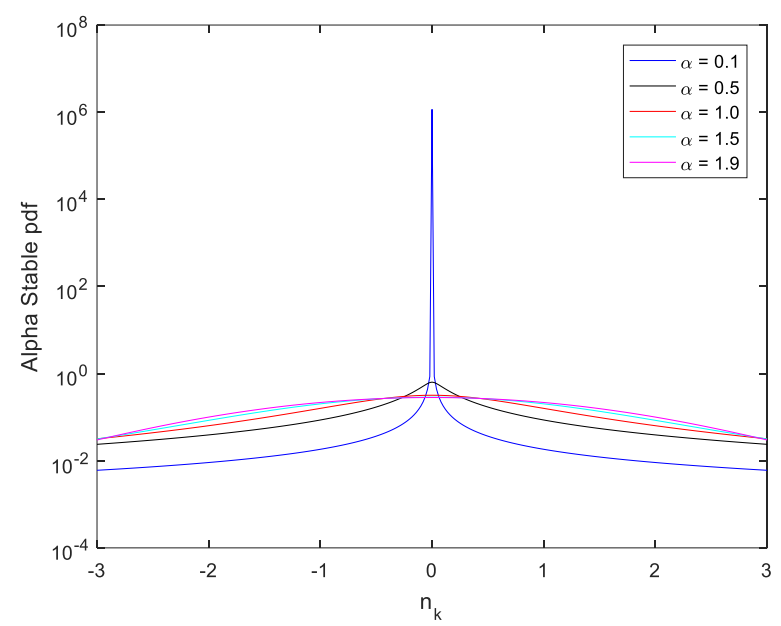

Fig. 16. Alpha stable pdf, $\beta=0, \gamma=1$ and $\delta=0$.

\section{CONCLUSIONS}

The three noise models, namely Middleton Class A, Bernoulli Gaussian and Alpha Stable models are used frequently in the literature for modeling the impulsive noise in PLC. In this study, we have investigated their probability density functions. Middleton Class A model has the advantage of selecting its parameters directly related to the physical channel. Further, the number of terms can be selected to be larger than two, which results with a more complicated but skilled model. It may model wider kind of date more precisely. Bernoulli Gaussian noise model is a multimodal distribution with two components. It can also model a wide range of data and its distribution is very close to Middleton Class A model, especially for heavy PLC impulsive noise case. Because of its simple form, Bernoulli Gaussian model can be preferable when calculation of analytic results is leading. Although it is not as powerful as the others, Alpha Stable model can also be used for impulse noise because of heavy tails. But it has a failure of having no closed form expression for its pdf. This is a handicap for analytic solutions.

\section{REFERENCES}

[1] G. Laguna-Sanchez, M. Lopez-Guerrero, "On the use of Alpha-Stable distributions in noise modeling for PLC", IEEE Transactions on Power Delivery, Vol.30, No.4, 2015, pp.1863-1870.

[2] T. Shongwe, A.J.H. Vinck, H.C. Ferreira, "A study on impulse noise and its models", SAIEE Africa Research Journal, Vol.106, No.3, 2015, pp.119-131.

[3] H. Chaouche, F. Gauthier, A. Zeddam, M. Tlich, M. Machmoum, "Time domain modeling of powerline impulsive noise at its source", Journal of Electromagnetic Analysis and Applications, Vol.3, No.9, 2011, pp.359367.

[4] T. Guzel, E. Ustunel, H.B. Celebi, H. Delic, K. Mihcak, "Noise Modeling and OFDM Receiver Design in Power-Line Communication", IEEE Transactions on Power Delivery, Vol.26, No.4, 2011, pp.27352742.

[5] Y. Himeur, A. Boukabou, "An adaptive recursive noise compensator for impulsive noise mitigation over OFDM power line communication", AEUE - International Journal of Electronics and Communications, Vol.70, No.1, 2016, pp.105-112. 
[6] G. Ndo, P. Siohan, M.H. Hamon, "Adaptive noise mitigation in impulsive environment: application to Power-Line Communications", IEEE Transactions on Power Delivery, Vol.25, No.2, 2010, pp.647-656.

[7] J. Lin, M. Nassar, B.L. Evans, "Impulsive noise mitigation in Powerline Communications using Sparse Bayesian learning", IEEE Journal on Selected Areas in Communications, Vol.31, No.7, 2013, pp.1172-1183.

[8] J.A. Cortes, A. Sanz, P. Estopinan, J.I. Garcia, "On the suitability of the Middleton class A noise model for Narrowband PLC", International Symposium on Power Line Communications and its Applications, 2016.

[9] H. Meng, Y.L. Guan, S. Chen, "Modeling and analysis of noise effects on broadband power-line communications", IEEE Transactions on Power Delivery, Vol.20, No.2, 2005, pp.630-637.

[10] F. Rouissi, H. Gassara, A. Ghazel, S. Najjar, "Comparative study of impulse noise models in the narrow band indoor PLC environment", 10th Workshop on Power Line Communications, 2016.

[11] Z. Zhang, Y. Sun, D. Wang, W. Xie, J. Liu, Y. Kuang, H. Ou, Q. Wang, Q. Lai, H.Y. Wang, B. Xu, Z. Liu, "Modeling and research of noise characteristics for low voltage Power Line Channel in OFDM communication system", 9th International Conference on Intelligent Human-Machine Systems and Cybernetics, 2017, pp.116-120.

[12] M. Katayama, T. Yamazato, H. Okada, "A mathematical model of noise in narrowband power line communication systems", IEEE Journal on Selected Areas in Communications, Vol.24, No.7, 2006, pp.1267-1276.

[13] N. Andreadou, F.N. Pavlidou, "Modeling the noise on the OFDM Power-Line Communications system", IEEE Transactions on Power Delivery, Vol.25, No.1, 2010, pp.150-157.

[14] F. Gianaroli, F. Pancaldi, G.M. Vitetta, "The impact of statistical noise modeling on the error-rate performance of OFDM Power-Line Communications", IEEE Transactions on Power Delivery, Vol.29, No.6, 2014, pp.2622-2630.

[15] M.A. Sonmez, M.Bagriyanik, "Statistical model study for Narrowband Power Line Communication Noises", 8th International Conference on Electrical and Electronics Engineering, 2013, pp.539-543.

[16] K.M. Rabie, E. Alsusa, "Improved DPTE technique for impulsive noise mitigation over power-line communication channels", AEUE International Journal of Electronics and Communications, Vol.69, No.12, 2015, pp.1847-1853.

\section{BIOGR APHIES}

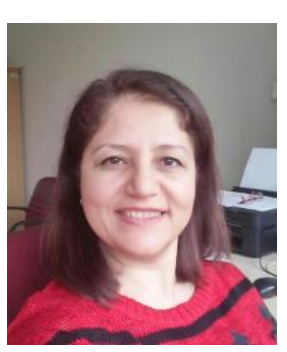

SELVA MURATOĞLU ÇÜRÜK received the B.S. degree from the Electrical and Electronics Engineering Department, Middle East Technical University (METU), Ankara, Turkey, in 1996, and the M.S. and Ph.D. degrees from the same university, in 1999 and 2008, respectively. She is currently at the Iskenderun Technical University.

Her research interests are wireless communication systems, digital communication theory, digital signal processing, multicarrier modulation and channel modeling. 